\title{
Model-Based Inference of Synaptic Transmission
}

\begin{abstract}
Ola Bykowska ${ }^{1}$, Camille Gontier ${ }^{2}$, Anne-Lene Sax ${ }^{1}$, David W. Jia ${ }^{3}$, Milton Llera Montero ${ }^{1,4}$, Alex D. Bird ${ }^{5,6}$, Conor Houghton ${ }^{1}$, Jean-Pascal Pfister ${ }^{2,7}$ and Rui Ponte Costa ${ }^{1,2 *}$

${ }^{1}$ Computational Neuroscience Unit, Department of Computer Science, SCEEM, Faculty of Engineering, University of Bristol, Bristol, United Kingdom, ${ }^{2}$ Department of Physiology, University of Bern, Bern, Switzerland, ${ }^{3}$ Department of Physiology, Anatomy and Genetics, Centre for Neural Circuits and Behaviour, University of Oxford, Oxford, United Kingdom, ${ }^{4}$ School of Psychological Science, Faculty of Life Sciences, University of Bristol, Bristol, United Kingdom, ${ }^{5}$ Ernst Strungmann Institute for Neuroscience in Cooperation With Max Planck Society, Frankfurt, Germany, ${ }^{6}$ Frankfurt Institute for Advanced Studies, Frankfurt, Germany, ${ }^{7}$ Institute of Neuroinformatics and Neuroscience Center Zurich, University of Zurich/ETH Zurich, Zurich, Switzerland
\end{abstract}

Synaptic computation is believed to underlie many forms of animal behavior. A correct identification of synaptic transmission properties is thus crucial for a better understanding of how the brain processes information, stores memories and learns. Recently, a number of new statistical methods for inferring synaptic transmission parameters have been introduced. Here we review and contrast these developments, with a focus on methods aimed at inferring both synaptic release statistics and synaptic dynamics. Furthermore, based on recent proposals we discuss how such methods can be applied to data across different levels of investigation: from intracellular paired experiments to in vivo network-wide recordings. Overall, these developments open the window to reliably estimating synaptic parameters in behaving animals.

Keywords: synaptic transmission, short-term synaptic plasticity, model inference, probabilistic inference, quantal analysis

\section{INTRODUCTION}

Modifications of synaptic transmission properties are believed to underlie learning, memory and, more generally, neural dynamics (Nabavi et al., 2014; Costa et al., 2017; Roelfsema and Holtmaat, 2018; Williams and Holtmaat, 2018; Llera-Montero et al., 2019). It is therefore of great importance to accurately infer synaptic transmission properties. Two key features that define synaptic communication are: stochastic transmission (Malagon et al., 2016) and (relatively fast) temporal dynamics (Markram et al., 1998; Zucker and Regehr, 2002). The former is reflected as trial to trial variability of synaptic transmission as the combined result of pre- and postsynaptic sources of noise, such as probabilistic vesicle release (presynaptic) or binding of quantal neurotransmitter packets to (postsynaptic) receptors (Faber and Korn, 1991; Traynelis et al., 1993). Whereas temporal dynamics is reflected in the temporal modulation of synaptic responses, which is mediated by the multiple time constants of the synaptic transmission machinery. Such dynamics give rise to the commonly observed phenomenon of short-term plasticity (STP) (Tsodyks and Markram, 1997; Zucker and Regehr, 2002). In this review we summarize, discuss and contrast recent developments in inference methods that capture either of these two elements (i.e., stochastic release and STP), or both. In particular our review focus on relatively simple phenomenological and statistical models, which abstract out the underlying biophysics and do not capture some aspects of synaptic transmission.

We also highlight recent advances toward inferring synaptic properties in vivo. Studying synaptic transmission parameters under naturalistic conditions is not only likely to give more precise parameters estimates, but also insights into what synaptic transmission properties are relevant in behaving animals (Dobrunz and Stevens, 1999; Isaac et al., 2009). 


\section{INFERENCE OF STOCHASTIC TRANSMISSION}

Synaptic transmission is inherently stochastic (see Figure 1 for a schematic). In the quantal view of synaptic transmission neurotransmitter-containing vesicles (quanta) are released into the synaptic cleft from $N$ release sites with probability $P_{\text {rel }}$ (Del Castillo and Katz, 1954; Korn and Faber, 1991; Larkman et al., 1991; Lanore and Silver, 2016) (Figure 1A). Once released, neurotransmitters bind to postsynaptic receptors triggering a postsynaptic response with mean quantal amplitude q. A binomial model is often used to describe these three aspects (i.e., number of release sites $N$, release probability $P_{\text {rel }}$ and the mean quantal amplitude $q$ ). In this model the mean peak of postsynaptic responses is given by $\mu=q N P_{\text {rel }}$ and their variance by $\sigma^{2}=q^{2} N P_{\text {rel }}\left(1-P_{\text {rel }}\right)$ (Figure 1B $)^{1}$. Several methods based on the binomial release model have been proposed to infer synaptic transmission parameters. A simple method relies solely on using the mean and variance to get estimates of both $q$ and $P_{\text {rel }}$ by rearranging the terms as $\hat{q}=\frac{\sigma^{2}}{\mu}+\frac{\mu}{N}$ and $\hat{P}_{\text {rel }}=\frac{\mu}{N \hat{q}}$ given a number of release sites $N$ (Markram et al., 1997; Costa et al., 2015). The variance-mean analysis (also known as multipleprobability fluctuation analysis) is a slightly more advanced technique that relies on recording postsynaptic responses under different release probability conditions, which are typical set experimentally by varying the concentration of extracellular calcium. The relationship between the variance and the mean (i.e., $\mu$ and $\sigma^{2}$ as above) under different release probabilities is then fitted to the parabolic function given by the binomial model (Figure 3A). This method estimates all three parameters $\left(N, P_{\text {rel }}\right.$, and $q$; see Lanore and Silver, 2016 for a detailed review on the topic). Because this method depends on having an accurate estimation of mean and variance of the postsynaptic responses, it requires relatively long and stable electrophysiological recordings under different conditions.

The binomial model described above may suffer from identifiability issues. For example, in the presence of a high level of noise it may not be possible to reliably separate the multiple peaks of the postsynaptic responses. In this case a simple Gaussian description of the synaptic responses may be preferable (Figure 2A). In addition, the methods described above also rely on point estimates which may lead to inaccurate conclusions due to correlations in the parameters (see Figure 2A for an example of such a case). A more principled approach to the problem that explicitly represents the uncertainty in the parameters should offer a better understanding of how well a particular model explains a given dataset (see section 3 for examples of this).

Building on earlier work (Turner and West, 1993), Bhumbra and Beato (2013) introduced a more principled quantal analysis method-Bayesian Quantal Analysis (BQA). This method applies Bayesian statistics which allows model inference to combine prior knowledge $P(\theta)$ over model parameters $\theta$ (e.g, $\left.\theta=\left(P_{r e l}, N, q\right)\right)$ with the data likelihood $P(D \mid \theta)$ following

\footnotetext{
${ }^{1}$ The binomial release model makes a few assumptions, namely that each site releases vesicles independently and that $P_{\text {rel }}$ is the same across different release sites.
}

Bayes' theorem as $P(\theta \mid D) \propto P(\theta) P(D \mid \theta)$. In contrast to standard optimization methods, Bayesian inference explicitly models uncertainty over parameters given prior knowledge. Choosing the appropriate prior is an important step when developing Bayesian frameworks as it shapes the posterior distribution over parameters given by the likelihood. In BQA, the prior is used to integrate a priori knowledge about the synaptic release statistics (e.g., expected bounds), which simultaneously models the distributions of postsynaptic responses recorded under multiple release probabilities (independent of each other). This is in contrast with standard mean-variance analysis described above, which simply models the mean responses across different release probabilities. By incorporating prior information, this method improves the accuracy of parameter inference and, importantly, reduces the number of samples needed compared to the mean-variance analysis (from about 100 samples to about 60 samples). Therefore, this new method may be preferable in experimental conditions where long recordings are particularly challenging (see a more detailed comparison in Table 1).

\section{INFERENCE OF SHORT-TERM PLASTICITY}

Postsynaptic responses are dynamic-the peak response amplitude depends not only on the quantal parameters, but also on previous activity. If the presynaptic neuron fires in quick succession, the released vesicles are not given enough time to be recycled, which leads to less vesicles available for release. As a consequence synaptic responses become weaker, also known as short-term depression (Figure 1A) and such recovery rates are often modeled with an exponential with timeconstant $\tau_{D}$. At the same time the presynaptic calcium levels can increase with every consecutive spike, which may lead to an increase in the postsynaptic response rather than a decrease-this is known as short-term facilitation.

\subsection{Deterministic Models of Short-Term Plasticity}

A number of deterministic short-term plasticity models have been proposed that characterize the dynamic properties of synaptic transmission (for a review on STP models see Hennig, 2013). These models capture STP data relatively well, and thus may enable us to uncover how STP may be regulated under different conditions.

The parameters of these models are commonly fit using leastsquares optimization to obtain a single set of parameters (point estimates) where the goal is to find the best (or at least a good) set of parameters that captures a given experimental dataset (Markram et al., 1998; Le Bé and Markram, 2006; Markram, 2006; Wang et al., 2006; Rinaldi et al., 2008; Ramaswamy et al., 2012; Testa-Silva et al., 2012; Romani et al., 2013) (Figure 3B).

However, estimating parameters of STP models poses a challenge. Similar to the issues highlighted above for binomial models, in most STP models different parameter sets produce model outputs that follow the observed data equally well 


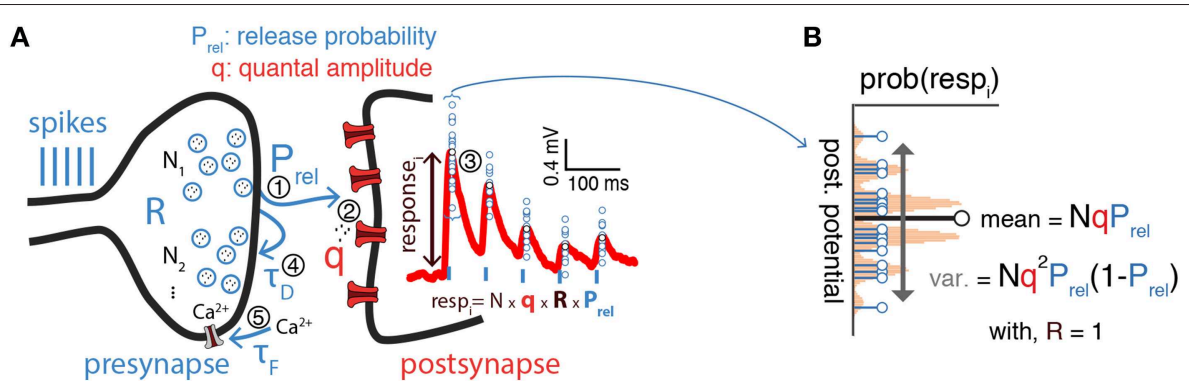

FIGURE 1 | Inference of synaptic transmission parameters. (A) Schematic of synaptic transmission parameters. On the left the different elements of the synaptic transmission process are represented: first (1), presynaptic spikes (blue vertical bars) lead to release of vesicles containing neurotransmitter ( $R$, for presynaptic resources) from one of $N$ possible release sites with probability $P_{\text {rel }}$; second (2), released neurotransmitters (quanta) bind to postsynaptic receptors triggering a response with amplitude q; third (3), this process triggers a postsynaptic response with average amplitude $N q R P_{\text {rel }}$, which takes into account both binomial and short-term synaptic plasticity; fourth (4), presynaptic vesicles are recovered with a time constant $\tau_{D}$ which may lead to short-term depression of consecutive postsynaptic responses (red trace on the postsynapse) before the presynaptic resources, $R$, fully recover; fifth (5), at the same time presynaptic voltage-dependent calcium $\left(\mathrm{Ca}^{2+}\right)$ channels can lead to calcium build-up on the presynapse (modeled by a time constant $\left.\tau_{F}\right)$, which may increase release probability $\left(P_{\text {rell }}\right)$ and in turn lead to an increase of consecutive postsynaptic responses, also known as short-term facilitation (not shown). (B) Postsynaptic responses exhibit variability [blue circles from (A) overlaid on top of the mean postsynaptic response in red]. Such variability is often described as a simple binomial process, with $N$ release sites and variance given by $N q^{2} P_{\text {rel }}\left(1-P_{\text {rel }}\right)$. Plot represents a binomial release model with $N=5, P_{\text {rel }}=0.5$ and some arbitrary $q$.

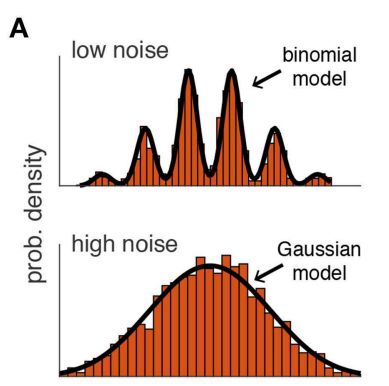

post. response

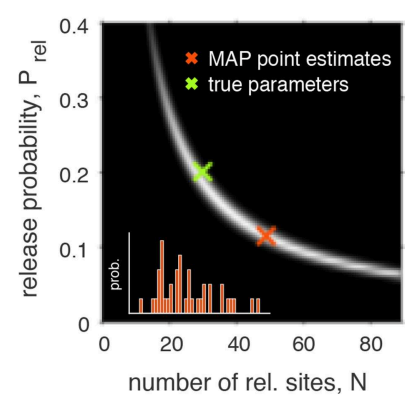

number of rel. sites, $\mathrm{N}$

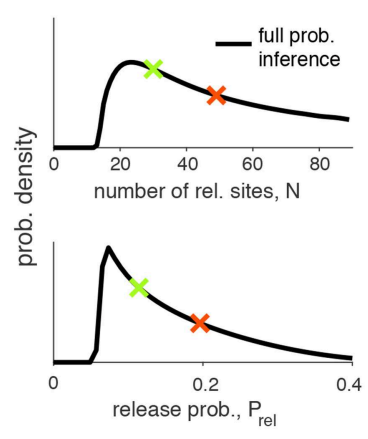

B

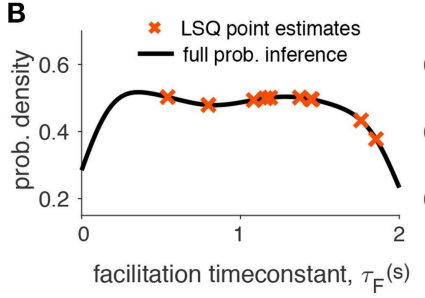

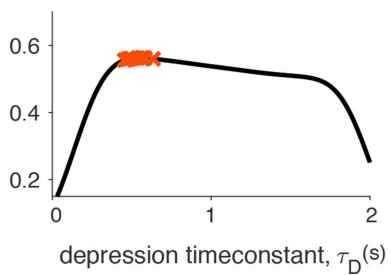

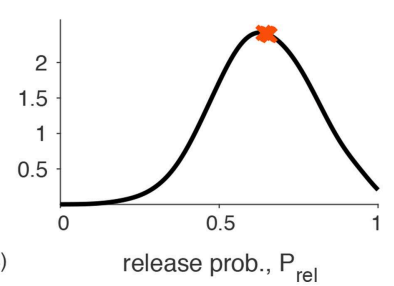

FIGURE 2 | Identifiability of synaptic transmission parameters. (A) Identifiability issues of quantal release models. Left upper figure: Histogram of 2000 simulated postsynaptic responses with $N=5, P_{\text {rel }}=0.5, q=1, \sigma=0.3$. In this case it is possible to fit a binomial model. Left lower figure: same simulation, but for high noise $(\sigma=0.7)$. The quantal peaks (i.e., the parameter $\mathrm{N}$ ) are not identifiable anymore if the recording noise is too high, and in this case a Gaussian model provides a better description of the synaptic responses. Middle panel: Pairwise posterior marginal for $N$ and $P_{\text {rel }}$ for a typical experimental case with 40 observations (simulated postsynaptic responses shown in inset) where the true parameters were $N=30, P_{\text {rel }}=0.2$ and $q=1$ (green cross). The maximum a posterior (MAP) estimates is obtained for $N=49$ and $P_{\text {rel }}=0.11$ (red cross): as $N$ and $p$ are anticorrelated, the posterior is roughly the same over a long band were $N$ and $p$ can be substituted, leading to inference error for a small number of observations. Right panel: Marginal posterior for $N$ and $P_{\text {rel }}$ from the previous panel. (B) Identifiability issues of short-term synaptic plasticity models. Given experimental data it is often of interest to infer the synaptic parameters. Two main types of inference have been applied: point estimations where a single scalar is estimated for one or more parameters (red crosses) or full probabilistic inference, where the full probability density over the parameters is obtained (black line). This particular example was obtained by inferring the Tsodyks-Markram model with four parameters given short-term plasticity recordings between pyramidal cells in layer-5 visual cortex (see Costa et al., 2013 for more details, only three parameters are shown here for simplicity: $\tau_{F}, \tau_{D}$ and $\left.P_{\text {rel }}\right)$. Point estimates were obtained using a standard least-square (LSQ) fitting method (simulated annealing). Full probabilistic inference was done using MCMC sampling following Costa et al. (2013) (see main text for more details). As demonstrated by Costa et al. (2013) the uncertainty over the parameters can be greatly reduced by using more informative protocols that cover a wider frequency range. 
TABLE 1 | Comparison of different model-based approaches.

\begin{tabular}{|c|c|c|c|c|c|}
\hline Approach & Binomial & STP & $\begin{array}{l}\text { Inference } \\
\text { quality }^{\text {h }}\end{array}$ & $\begin{array}{c}\text { Experimental } \\
\text { ease }^{h}\end{array}$ & $\begin{array}{l}\text { Algorithm } \\
\text { complexity }\end{array}$ \\
\hline Mean-variance analysis ${ }^{a}$ & $\checkmark$ & $\times$ & ** & * (PSR) & $\mathcal{O}(M)$ \\
\hline Bayesian quantal analysis ${ }^{b}$ & $\checkmark$ & $\times$ & 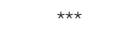 & ** (PSR) & $\mathcal{O}(M N)$ \\
\hline Least-square STP fitting ${ }^{C}$ & $\times$ & $\checkmark$ & ** & 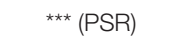 & $\mathcal{O}(M)$ \\
\hline Bayesian Gaussian-STPd & $\times$ & $\checkmark$ & 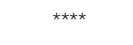 & ${ }^{\star \star \star}(\mathrm{PSR})$ & $\mathcal{O}(M S)$ \\
\hline Binomial-STPe & $\checkmark$ & $\checkmark$ & 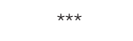 & ${ }^{\star \star \star}(\mathrm{PSR})$ & $\mathcal{O}\left(M N^{4}\right)$ \\
\hline Bayesian binomial-STPf & $\checkmark$ & $\checkmark$ & 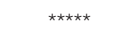 & *** (PSR) & $\mathcal{O}\left(M N^{4}\right)$ \\
\hline Spike-based GLMg & $\times$ & $\checkmark$ & * & 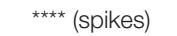 & $\mathcal{O}(M)$ \\
\hline
\end{tabular}

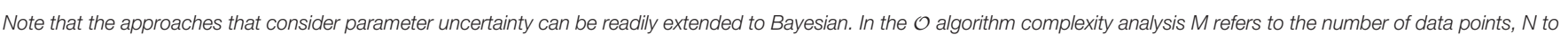

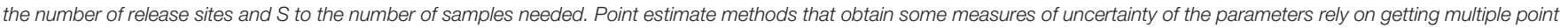

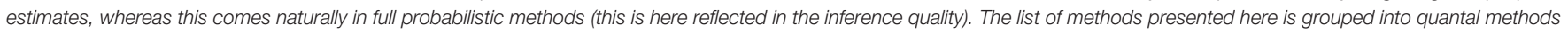

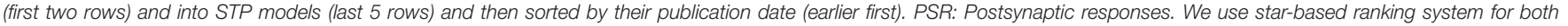
inference quality and experimental ease, where one star means worse/harder.

a see Korn and Faber (1991), Lanore and Silver (2016), and Figure 3A.

${ }^{b}$ see Bhumbra and Beato (2013) and Figure $3 \boldsymbol{A}$.

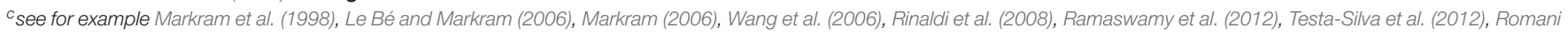
et al. (2013) and Figure 3B.

${ }^{d}$ see Costa et al. (2013) and Figure 3C.

esee Loebel et al. (2009), Barri et al. (2016), and Figure 3C.

${ }^{f}$ see Bird et al. (2016) and Figure 3C.

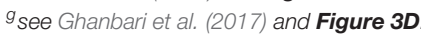

${ }^{h}$ Note that this ranking is subjective and based purely on our experience with these methods.

(Figure 2B; Costa et al., 2013). The existence of these multiple plausible solutions opens problems when relying on point estimates to draw conclusions about the underlying biological mechanisms. Therefore, it is important to also consider the uncertainty of the parameter estimation. Unlike single point estimate approaches, full probabilistic inference naturally captures parameter uncertainty, which enables a more comprehensive model comparison (e.g., Akaike Information Criterion, Bayesian Information Criterion or Bayes factor). Note that this can also be in principle obtained using sensitivity or cross-validation analysis when using standard fitting methods (Varela et al., 1997; Tennøe et al., 2018), but as highlighted in Figure 2B these methods may not provide a complete picture of the parameter landscape. One form of probabilistic inference is full Bayesian inference where, similar to the BQA approach, we aim to obtain the posterior distribution of STP parameters given experimentally observed data.

Costa et al. (2013) introduced the first Bayesian inference framework of STP models (Bayesian Gaussian-STP; Figure 3C; Table 1). In this work the authors modeled the mean postsynaptic peak responses using the Tsodyks-Markram STP model to account for the dynamic properties of the synapse (Tsodyks and Markram, 1997; Markram et al., 1998). The Tsodyks-Markram STP model is a commonly used model built around the synaptic dynamics discussed above. In order to capture the variability of synaptic responses, Costa et al. (2013) used a Gaussian approximation as the likelihood and a flat (uninformative) prior with reasonable bounds over the parameters. Calculating the posterior exactly is often intractable due to complex likelihoods and intractable normalizing constants. Instead, Costa et al. (2013) obtained the posterior distribution $P(\theta \mid D)$ via sampling using a Markov Chain Monte Carlo (MCMC) algorithm.
MCMC methods rely on constructing a Markov chain ${ }^{2}$ that should converge to the desired probability distribution in the equilibrium (i.e., after long enough observations).

This method was used to study the parameter uncertainty given datasets obtained with common experimental protocols, which are typically based on regular spike trains. The posterior distributions revealed that some of the parameters from the Tsodyks-Markram STP models were poorly constrained by such experimental protocols (Figure 2B). This observation led to the proposal of new and irregular experimental protocols that span a broader stimulation frequency range and result in substantially reduced uncertainty over the parameter values. Such protocols not only lead to reduced uncertainty, but can also be more easily applied in realistic and natural conditions (Dobrunz and Stevens, 1999).

Furthermore, obtaining the posterior distribution helps to understand the dependencies between parameters, which is not straightforward using traditional fitting methods. For example, in Stone et al. (2014), the authors used an MCMC method to obtain the posterior distribution over the parameters (similar to Costa et al., 2013) allowing the authors to highlight two strongly correlated parameters. Importantly, the identification of this correlation led to a reparameterization of the model which improved parameter inference. Therefore, obtaining the posterior distribution over the parameters makes it possible to characterize their uncertainty and explore possible dependencies between parameters. Such MCMC methods are relatively efficient as long as the model can be computed efficiently (up to a few seconds) and the number of STP parameters remains relatively low (less than a few dozens).

${ }^{2}$ A Markov chain represents a probabilistic transition between states, in which a given transition depends only on the previous state. 


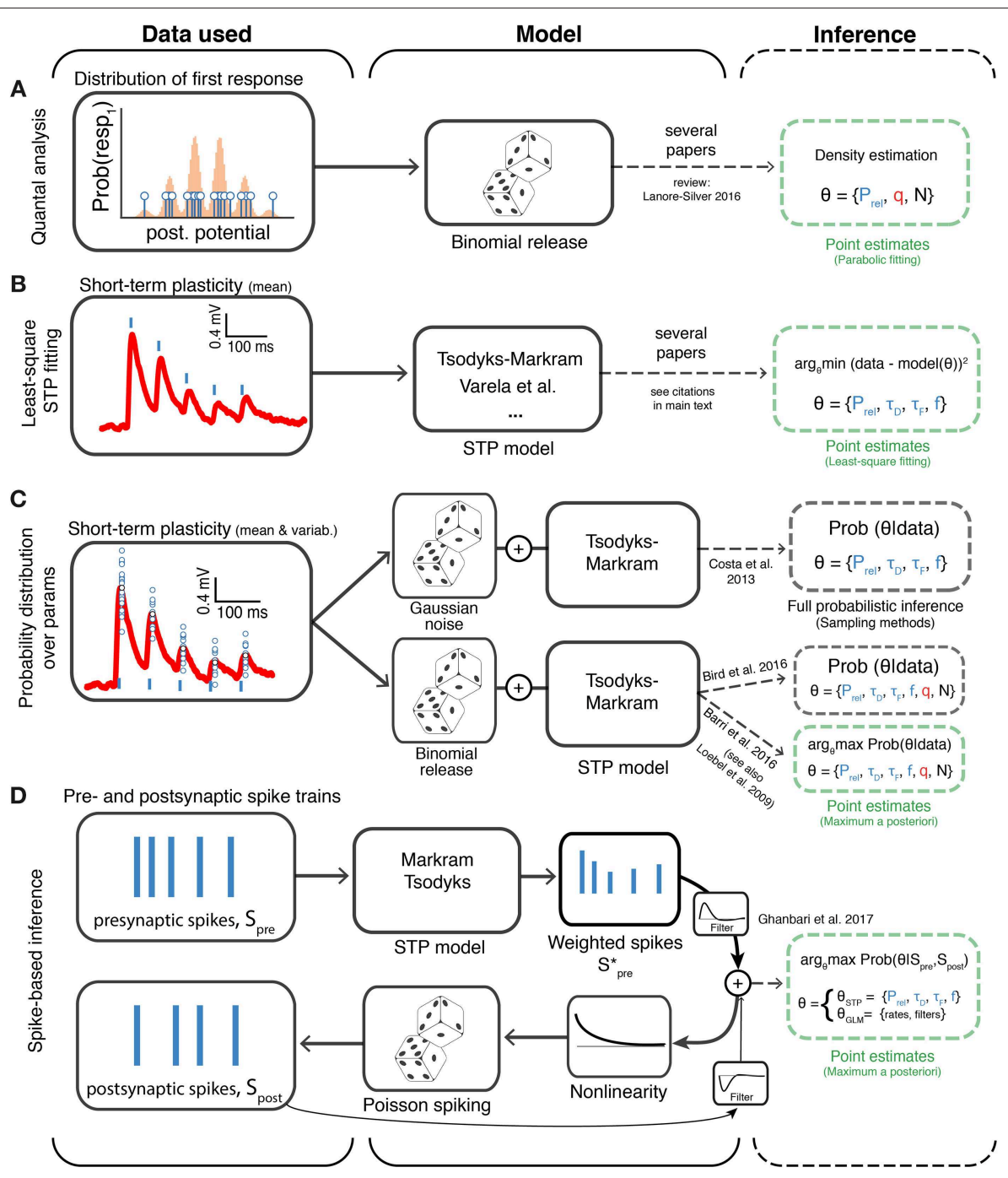

FIGURE 3 | Different approaches to model-based inference of synaptic transmission. The different methods are organized based on the type of experimental data to which they are applied (first column), the model being assumed (second column) and the method of parameter inference (third column; dashed green and gray boxes indicate point estimate and full probabilistic inference, respectively). (A) Methods that use the variability of the first postsynaptic responses to infer binomial release statistics. (B) Methods that rely on multiple averaged responses to fit short-term plasticity (STP) models, which typically discard binomial release statistics. (C) Methods that directly consider both variability and multiple synaptic responses using probability theory to infer the synaptic transmission parameters. Here two variants have been explored: (i) a Bayesian framework where Gaussian noise is used to model the synaptic response variability (Costa et al., 2013) and (ii) a framework in which binomial release statistics are explicitly considered (Barri et al., 2016; Bird et al., 2016). The later has been explored using two variants: full inference (using sampling, Bird et al., 2016) and optimization methods (Barri et al., 2016). (D) Methods that work directly at the level of spike trains and try to infer short-term plasticity parameters. Ghanbari et al. (2017) introduced a new method based on generalized linear models (GLMs) to obtain point estimates of short-term plasticity models. $P_{\text {rel }}$ : Release probability; $q$ : mean quantal amplitude; $N$ : number of release sites; $\tau_{D}$ : depression time constant; $\tau_{F}$ : facilitation time constant; $f$ : facilitation rate. Similarly to previous figures the mean postsynaptic responses are shown in red, spikes in blue (vertical lines) and small blue circles represent individual samples of postsynaptic responses.

\subsection{Stochastic Short-Term Plasticity Models}

There are two important limitations of relying on deterministic STP models. First, the optimization depends on an accurate estimation of the mean synaptic responses. As mentioned above, this requires a high number of trials, which is experimentally challenging (see Table 1). Second, by only considering averages these methods ignore the correlations between postsynaptic peaks, yet these correlations may provide valuable information to accurately infer the synaptic properties.

A couple of recent studies introduced methods that incorporate correlations between postsynaptic responses in the inference of STP parameters. These methods allow the extraction of both quantal and dynamic parameters of synaptic transmission from trains of postsynaptic responses without the requirement of averaging over multiple sweeps (Loebel 
et al., 2009; Barri et al., 2016; Bird et al., 2016). These studies implemented stochastic models of synaptic transmission by combining phenomenological Tsodyks-Markram STP models with binomial models of vesicular release and replenishment. The probability of vesicle release is derived from a TsodyksMarkram model and the vesicle replenishment probability is modeled with a Poisson process controlled by a depression time constant parameter $\tau_{D}$. The quantal size of the postsynaptic response evoked by each vesicle can be approximated by either a gamma distribution in Bird et al. (2016), or an inverse Gaussian distribution in Barri et al. (2016), and the total amplitude is modeled as a linear combination of these distributions. The choice of these distributions is motivated by the fact that the quantal amplitude distribution is positively skewed, a feature that can not be captured by a Gaussian distribution.

The full formulation of the stochastic STP model can be used to define the likelihood of the observed data given the model, $P(D \mid \theta)$. The stochasticity of the model introduces correlations between peaks in the train and these correlations pose the main difficulty in the likelihood calculation. In particular, because the amplitude of the postsynaptic response is dependent on the number of released vesicles. As discussed in Barri et al. (2016), if the likelihood is to be formulated using the probability distribution of released vesicles, the number of terms in the calculation would grow exponentially. This becomes a permutation with repetition problem, in order to account for correlations of released vesicles the number of terms in the calculation would grow as $(N+1)^{K}$ with $N$ being the number of release sites and $K$ corresponding to the number of spikes in the train. To make the calculation more efficient, in both studies the likelihood function is formulated in terms of the probability distributions of the release sites before and after a spike (rather than continuously), which fully captures the state of the system.

These two studies apply different strategies to obtain point estimates from the likelihood. Barri et al. (2016) uses an expectation-maximization algorithm (referred to as BinomialSTP in Table 1; see also Loebel et al., 2009), while Bird et al. (2016) uses MCMC sampling and flat priors (referred to as Bayesian binomial-STP in Table 1). While both methods return a point estimate of the parameter set that maximizes the likelihood function, only the sampling approach approximates the joint likelihood distribution of the parameters. As discussed above, by obtaining the full likelihood, not just a point estimate, Bird et al. (2016) explicitly quantifies the uncertainty over the parameters, and the full likelihood density (or posterior) can be analyzed. Moreover, it also allows for correlations between the distributions over the parameters to be studied.

The main features of these approaches are (i) accounting for correlations between subsequent postsynaptic responses and (ii) using individual postsynaptic traces for fitting the models, which offers theoretical and practical advantages. Interestingly, both Barri et al. (2016) and Bird et al. (2016) report that considering correlations during inference yields estimates of synaptic parameters that are more accurate and require less sweeps when compared to ignoring correlations. This means that the experimental protocols can be shorter, hence making these inference methods particularly attractive for experiments in vivo.

\section{TOWARD INFERENCE OF SYNAPTIC TRANSMISSION IN VIVO}

Recent developments have started to raise the possibility of accurately inferring synaptic transmission properties in vivo. One way to tackle this problem is to perform whole-cell recordings in vivo while stimulating the presynaptic neurons (or presenting a stimuli) (Costa et al., 2015; Pala and Petersen, 2015; SedighSarvestani et al., 2019). This is a valuable approach that is enabling the community to confirm previous in vitro results in vivo. For example, Costa et al. (2015) applied binomial-based estimation methods typically used in slices to in vivo data, and obtained results consistent with both modeling predictions and slice data. Puggioni et al. (2017) and Latimer et al. (2018) introduced new statistical methods with some success in inferring synaptic conductances from in vivo intracellular recordings and spike trains, respectively. However, these methods were not developed to estimate quantal or synaptic dynamics properties. In order to test how such synaptic features are shaped in more natural conditions across different brain regions new methods are required that can operate on the growing imaging-based or spike-based datasets.

Detecting synaptic connections from spikes alone is challenging. Even in the case of simple monosynaptic connections this is not straightforward (Fetz et al., 1991), but there have been recent successful attempts (English et al., 2017). One of the key difficulties in inferring synaptic parameters from spikes is that several non-synaptic variables can have an impact on the spiking statistics (Stevenson et al., 2008). For example, when a presynaptic neuron fires at high frequencies one would expect a reduction in the firing rate of the postsynaptic neuron due to short-term depression at their synaptic connections, but a similar effect can also be mediated by postsynaptic neuron-wide adaptation mechanisms (Brette and Gerstner, 2005).

A first attempt at tackling this problem has recently been put forward (Ghanbari et al., 2017). In this framework, the authors extended a generalized linear model to infer both neuronal and STP parameters directly from spike-trains (Figure 3D, referred to as spike-based GLM in Table 1). Interestingly, using their framework Ghanbari et al. (2017) showed that in a reduced system-a single postsynaptic neuron in slices with simulated inputs-postsynaptic adaptation can be distinguished from short-term depression as they are predominantly correlated with pre- and postsynaptic firing rates, respectively. More recently the same authors (Ghanbari et al., 2018) went further and used their framework to show that functional connectivity with STP may explain the diversity of activity patterns observed in vivo between different brain areas. However, for these approaches to provide accurate estimates of synaptic transmission properties (Table 1) in vivo many other factors need to be considered in future work, such as network dynamics, cell-type specificity and dendritic integration.

As highlighted above (section 3), inferring synaptic parameters using naturalistic conditions (e.g., spike patterns) not only is likely to give more precise estimates of synaptic parameters, but also insights into which synaptic transmission 
properties are relevant in behaving animals (Dobrunz and Stevens, 1999; Isaac et al., 2009).

\section{DISCUSSION}

In this review we have provided an overview of standard methods and recent developments of model-based inference of synaptic transmission. We started out by reviewing methods that rely on the binomial statistics of the first postsynaptic response alone and moved on to methods that consider the dynamics of consecutive synaptic responses (short-term plasticity) and their statistical properties. Historically, inference methods have mostly focused on point estimations, which give a biased interpretation of synaptic data (Costa et al., 2013). More recent developments have focused on full probabilistic inference, thus providing a more comprehensive picture on the most likely synaptic transmission parameters (Bhumbra and Beato, 2013; Costa et al., 2013; Bird et al., 2016).

One research direction that should improve the inference quality of the short-term plasticity parameters is to optimize the experimental protocol, namely the timings of the presynaptic action potentials. The stimulation protocol needs to be within some acceptable range (a too high stimulation frequency would induce long-term plasticity and thereby violate the stationarity assumption). However, within such a range, there is a lot of freedom that can be exploited to improve the quality of the parameter estimates. For example, Costa et al. (2013) explored a few different protocols (regular spike trains, regular spike train + recovery spike(s) or Poisson spike trains). It would be important to systematically study the space of protocols and determine which ones are the most informative. Pushing this idea even further, it would be interesting to design a closed-loop inference scheme such that after each spike and its subsequent postsynaptic response, the algorithm determines the best interval for the next spike that is maximally informative about the synaptic parameters.

In this review, we have not covered some other properties that are of interest. One that has received attention recently is the inference of the size of the presynaptic readily-releasable vesicle pool (Abrahamsson et al., 2017; Barros-Zulaica et al., 2019). Additionally, we have focused on the binomial release model, but many synapses require different release probabilities and quantal amplitudes across release sites, which is better captured by multinomial statistics (Walmsley et al., 1988; Lanore and Silver, 2016). There are several other important aspects of synaptic transmissoin not considered here, such as constrains on trial-to-trial quantal variability (Kullmann, 1993), STP models that also account for changes in quantal amplitude

\section{REFERENCES}

Abrahamsson, T., Chou, C. Y. C., Li, S. Y., Mancino, A., Costa, R. P., Brock, J. A., et al. (2017). Differential regulation of evoked and spontaneous release by presynaptic NMDA receptors. Neuron 96, 839-855. doi: 10.1016/j.neuron.2017.09.030
(Scheuss et al., 2002), frequency-dependent recovery rates in STP (Fuhrmann et al., 2004), and release-independent short-term depression (Bellingham and Walmsley, 1999; Fuhrmann et al., 2004). In future work, it would be important to understand how the developments reviewed here can also consider and be used to better understand these finer aspects of synaptic transmission.

There have been remarkable developments in measuring synaptic properties with high temporal and spatial resolution (Rey et al., 2015; Tang et al., 2016). Of particular interest are recent advances in ultrafast optical glutamate sensors, which are enabling measurements of synaptic release with high accuracy (Helassa et al., 2018). These developments, when coupled with the statistical inference frameworks reviewed here (Costa et al., 2013; Bird et al., 2016; Ghanbari et al., 2017, but see also Soares et al., 2019), raise the possibility of accurate optical estimation of synaptic transmission properties in awake behaving animals.

Finally, there has been a recent surge in new and exciting large-scale recordings, such as voltage and calcium imaging (Piatkevich et al., 2019), multi-patch recordings (Peng et al., 2019) and multi-electrode spike recordings (Jun et al., 2017). With such methods at hand now is the right time to start asking questions that bridge systems neuroscience and synaptic transmission properties. By building on initial studies on how synapses are shaped by naturalistic spike-trains (Dobrunz and Stevens, 1999; Isaac et al., 2009), this body of work opens the possibility of inferring quantal and dynamic properties of synapses over multiple brain areas as animals learn a particular task.

Taken together these novel inference and experimental methods open the possibility of testing different theories put forward for the role of synaptic transmission in learning and memory (Pfister et al., 2010; Costa et al., 2015, 2017; LleraMontero et al., 2019), but also their impact in pathological states (Jackson et al., 2017).

\section{AUTHOR CONTRIBUTIONS}

$\mathrm{OB}, \mathrm{CG}$, and RC generated the figures. OB, CG, A-LS, DJ, MM, $\mathrm{AB}, \mathrm{CH}, \mathrm{J}-\mathrm{PP}$, and $\mathrm{RC}$ wrote the manuscript.

\section{ACKNOWLEDGMENTS}

We would like to thank Mark van Rossum and Magnus Richardson for useful feedback on this review. OB and A-LS work was supported by the Wellcome Trust Doctoral Training Programme in Neural Dynamics, Grant no. 105207/Z/14/Z and $215303 / \mathrm{Z} / 19 / \mathrm{Z}$, respectively. MM was supported by the European Research Council, grant no. 741134 to Jeff Bowers. 
size at synaptic connections in a neocortical microcircuit. bioRxiv 646497. doi: $10.1101 / 646497$

Bellingham, M. C., and Walmsley, B. (1999). A novel presynaptic inhibitory mechanism underlies paired pulse depression at a fast central synapse. Neuron 23, 159-170. doi: 10.1016/S0896-6273(00)80762-X

Bhumbra, G. S., and Beato, M. (2013). Reliable evaluation of the quantal determinants of synaptic efficacy using Bayesian analysis. J. Neurophysiol. 109, 603-620. doi: 10.1152/jn.00528.2012

Bird, A. D., Wall, M. J., and Richardson, M. J. (2016). Bayesian inference of synaptic quantal parameters from correlated vesicle release. Front. Comput. Neurosci. 10:116. doi: 10.3389/fncom.2016.00116

Brette, R., and Gerstner, W. (2005). Adaptive exponential integrate-and-fire model as an effective description of neuronal activity. J. Neurophysiol. 94:3637. doi: 10.1152/jn.00686.2005

Costa, R. P., Froemke, R. C., Sjostrom, P. J., and van Rossum, M. C. W. (2015). Unified pre- and postsynaptic long-term plasticity enables reliable and flexible learning. eLife 4:e09457. doi: 10.7554/eLife.11988

Costa, R. P., Padamsey, Z., D’amour, J. A., Emptage, N. J., Froemke, R. C., and Vogels, T. P. (2017). Synaptic transmission optimization predicts expression loci of long-term plasticity. Neuron 96, 177-189.e7. doi: 10.1016/j.neuron.2017.09.021

Costa, R. P., Sjöström, P. J., and van Rossum, M. C. W. (2013). Probabilistic inference of short-term synaptic plasticity in neocortical microcircuits. Front. Comput. Neurosci. 7:75. doi: 10.3389/fncom.2013.00075

Del Castillo, J., and Katz, B. (1954). Quantal components of the end-plate potential. J. Physiol. 124, 560-573. doi: 10.1113/jphysiol.1954.sp005129

Dobrunz, L. E., and Stevens, C. F. (1999). Response of hippocampal synapses to natural stimulation patterns. Neuron 22, 157-166. doi: 10.1016/S0896-6273(00)80687-X

English, D. F., McKenzie, S., Evans, T., Kim, K., Yoon, E., and Buzsáki, G. (2017). Pyramidal cell-interneuron circuit architecture and dynamics in hippocampal networks. Neuron 96, 505-520.e7. doi: 10.1016/j.neuron.2017.09.033

Faber, D. S., and Korn, H. (1991). Applicability of the coefficient of variation method for analyzing synaptic plasticity. Biophys. J. 60, 1288-1294. doi: 10.1016/S0006-3495(91)82162-2

Fetz, E., Toyama, K., and Smith, W. (1991). "Synaptic interactions between cortical neurons," in Normal and Altered States of Function, eds A. Peters and E. J. Jones (Boston, MA: Springer), 1-47.

Fuhrmann, G., Cowan, A., Segev, I., Tsodyks, M., and Stricker, C. (2004). Multiple mechanisms govern the dynamics of depression at neocortical synapses of young rats. J. Physiol. 557(Pt 2):415-438. doi: 10.1113/jphysiol.2003.058107

Ghanbari, A., Malyshev, A., Volgushev, M., and Stevenson, I. H. (2017). Estimating short-term synaptic plasticity from pre- and postsynaptic spiking. PLoS Comput. Biol. 13:e1005738. doi: 10.1371/journal.pcbi.1005738

Ghanbari, A., Ren, N., Keine, C., Stoelzel, C., and Stevenson, I. H. (2018). Functional connectivity with short-term dynamics explains diverse patterns of excitatory spike transmission in vivo. bioRxiv. doi: 10.1101/475178

Helassa, N., Dürst, C. D., Coates, C., Kerruth, S., Arif, U., Schulze, C., et al. (2018). Ultrafast glutamate sensors resolve high-frequency release at Schaffer collateral synapses. Proc. Natl. Acad. Sci. U.S.A. 115, 5594-5599. doi: $10.1073 /$ pnas.1720648115

Hennig, M. H. (2013). Theoretical models of synaptic short term plasticity. Front. Comput. Neurosci. 7:45. doi: 10.3389/fncom.2013.00045

Isaac, J. T., Buchanan, K. A., Muller, R. U., and Mellor, J. R. (2009). Hippocampal place cell firing patterns can induce long-term synaptic plasticity in vitro. J. Neurosci. 29, 6840-6850. doi: 10.1523/JNEUROSCI.0731-09.2009

Jackson, J. S., Witton, J., Johnson, J. D., Ahmed, Z., Ward, M., Randall, A. D., et al. (2017). Altered synapse stability in the early stages of tauopathy. Cell Rep. 18, 3063-3068. doi: 10.1016/j.celrep.2017.03.013

Jun, J. J., Steinmetz, N. A., Siegle, J. H., Denman, D. J., Bauza, M., Barbarits, B., et al. (2017). Fully integrated silicon probes for high-density recording of neural activity. Nature 551, 232-236. doi: 10.1038/nature24636

Korn, H., and Faber, D. S. (1991). Quantal analysis and synaptic efficacy in the CNS. Trends Neurosci. 14, 439-445. doi: 10.1016/0166-2236(91)90042-S

Kullmann, D. M. (1993). Quantal variability of excitatory transmission in the hippocampus: implications for the opening probability of fast glutamate-gated channels. Proc. Biol. Sci. 253, 107-116. doi: 10.1098/rspb.1993.0088
Lanore, F., and Silver, R. A. (2016). "Extracting quantal properties of transmission at central synapses," in Advanced Patch-Clamp Analysis for Neuroscientists, ed A. Korngreen (New York, NY: Springer), 193-211.

Larkman, A., Stratford, K., and Jack, J. (1991). Quantal analysis of excitatory synaptic action and depression in hippocampal slices. Nature 350, 344-347. doi: $10.1038 / 350344 \mathrm{a} 0$

Latimer, K., Rieke, F., and Pillow, J. W. (2018). Inferring synaptic inputs from spikes with a conductance-based neural encoding model. bioRxiv 281089. doi: 10.1101/281089

Le Bé, J., and Markram, H. (2006). Spontaneous and evoked synaptic rewiring in the neonatal neocortex. Proc. Natl. Acad. Sci. U.S.A. 103:13214. doi: 10.1073/pnas.0604691103

Llera-Montero, M., Sacramento, J., and Costa, R. P. (2019). Computational roles of plastic probabilistic synapses. Curr. Opin. Neurobiol. 54, 90-97. doi: 10.1016/j.conb.2018. 09.002

Loebel, A., Silberberg, G., Helbig, D., Markram, H., Tsodyks, M., and Richardson, M. J. E. (2009). Multiquantal release underlies the distribution of synaptic efficacies in the neocortex. Front. Comput. Neurosci. 3:27. doi: 10.3389/neuro.10.027.2009

Malagon, G., Miki, T., Llano, I., Neher, E., and Marty, A. (2016). Counting vesicular release events reveals binomial release statistics at single glutamatergic synapses. J. Neurosci. 36, 4010-4025. doi: 10.1523/JNEUROSCI.4352-15.2016

Markram, H. (2006). The blue brain project. Nat. Rev. Neurosci. 7, 153-160. doi: $10.1038 / \mathrm{nrn} 1848$

Markram, H., Lübke, J., Frotscher, M., Roth, A., and Sakmann, B. (1997). Physiology and anatomy of synaptic connections between thick tufted pyramidal neurones in the developing rat neocortex. J. Physiol. 500(Pt 2):409440. doi: 10.1113/jphysiol.1997.sp022031

Markram, H., Wang, Y., and Tsodyks, M. (1998). Differential signaling via the same axon of neocortical pyramidal neurons. Proc. Natl. Acad. Sci. U.S.A. 95, 5323-5328. doi: 10.1073/pnas.95.9.5323

Nabavi, S., Fox, R., Proulx, C. D., Lin, J. Y., and Tsien, R. Y. (2014). Engineering a memory with LTD and LTP. Nature. 511:348. doi: 10.1038/nature13294

Pala, A., and Petersen, C. C. H. (2015). In vivo measurement of celltype-specific synaptic connectivity and Synaptic transmission in layer 2/3 mouse barrel cortex. Neuron 85, 68-75. doi: 10.1016/j.neuron.2014. 11.025

Peng, Y., Mittermaier, F. X., Planert, H., Schneider, U. C., Alle, H., and Geiger, J. R. P. (2019). High-throughput microcircuit analysis of individual human brains through next-generation multineuron patch-clamp. bioRxiv 284:639328. doi: $10.1101 / 639328$

Pfister, J. P., Dayan, P., and Lengyel, M. (2010). Synapses with short-term plasticity are optimal estimators of presynaptic membrane potentials. Nat. Neurosci. 13, 1271-1275. doi: 10.1038/nn.2640

Piatkevich, K. D., Bensussen, S., Tseng, H.-A., Shroff, S. N., Lopez-Huerta, V. G., Park, D., et al. (2019). Population imaging of neural activity in awake behaving mice in multiple brain regions. bioRxiv 350:616094. doi: 10.1101/616094

Puggioni, P., Jelitai, M., Duguid, I., and van Rossum, M. C. W. (2017). Extraction of synaptic input properties in vivo. Neural Comput. 29, 1745-1768. doi: 10.1162/NECO_a_00975

Ramaswamy, S., Hill, S. L., King, J. G., Schürmann, F., Wang, Y., and Markram, H. (2012). Intrinsic morphological diversity of thick-tufted layer 5 pyramidal neurons ensures robust and invariant properties of in silico synaptic connections. J. Physiol. 590, 737-752. doi: 10.1113/jphysiol.2011.219576

Rey, S. A., Smith, C. A., Fowler, M. W., Crawford, F., Burden, J. J., and Staras, K. (2015). Ultrastructural and functional fate of recycled vesicles in hippocampal synapses. Nat. Commun. 6:8043. doi: 10.1038/ncomms9043

Rinaldi, T., Silberberg, G., and Markram, H. (2008). Hyperconnectivity of local neocortical microcircuitry induced by prenatal exposure to valproic acid. Cereb. Cortex 18, 763-770. doi: 10.1093/cercor/bhm117

Roelfsema, P. R., and Holtmaat, A. (2018). Control of synaptic plasticity in deep cortical networks. Nat. Rev. Neurosci. 19, 166-180. doi: 10.1038/nrn.2018.6

Romani, A., Marchetti, C., Bianchi, D., Leinekugel, X., Poirazi, P., Migliore, M., et al. (2013). Computational modeling of the effects of amyloid-beta on release probability at hippocampal synapses. Front. Comput. Neurosci. 7:1. doi: 10.3389/fncom.2013.00001 
Scheuss, V., Schneggenburger, R., and Neher, E. (2002). Separation of presynaptic and postsynaptic contributions to depression by covariance analysis of successive EPSCs at the calyx of held synapse. J. Neurosci. 22, 728-739. doi: 10.1523/JNEUROSCI.22-03-00728.2002

Sedigh-Sarvestani, M., Palmer, L. A., and Contreras, D. (2019). Thalamocortical synapses in the cat visual system in vivo are weak and unreliable. eLife 8:e41925. doi: $10.7554 /$ eLife.41925

Soares, 1 C., Trotter, D., Longtin, A., Béïque, J.-C., and Naud, R. (2019). Parsing out the variability of transmission at central synapses using optical quantal analysis. bioRxiv 10:624692. doi: 10.1101/624692

Stevenson, I. H., Rebesco, J. M., Miller, L. E., and Kording, K. P. (2008). Inferring functional connections between neurons. Curr. Opin. Neurobiol. 18, 582-588. doi: 10.1016/j.conb.2008.11.005

Stone, E., Haario, H., and Lawrence, J. J. (2014). A kinetic model for the frequency dependence of cholinergic modulation at hippocampal GABAergic synapses. Math. Biosci. 258, 162-175. doi: 10.1016/j.mbs.2014.09.013

Tang, A. H., Chen, H., Li, T. P., Metzbower, S. R., MacGillavry, H. D., and Blanpied, T. A. (2016). A trans-synaptic nanocolumn aligns neurotransmitter release to receptors. Nature 536, 210-214. doi: 10.1038/nature19058

Tennøe, S., Halnes, G., and Einevoll, G. T. (2018). Uncertainpy: a python toolbox for uncertainty quantification and sensitivity analysis in computational neuroscience. Front. Neuroinformat. 12:e94. doi: 10.3389/fninf.2018.00049

Testa-Silva, G., Loebel, A., Giugliano, M., de Kock, C. P. J., Mansvelder, H. D., and Meredith, R. M. (2012). Hyperconnectivity and slow synapses during early development of medial prefrontal cortex in a mouse model for mental retardation and autism. Cereb. Cortex 22, 1333-1342. doi: 10.1093/cercor/bhr224

Traynelis, S. F., Silver, R. A., and Cull-Candy, S. G. (1993). Estimated conductance of glutamate receptor channels activated during EPSCs at the cerebellar mossy fiber-granule cell synapse. Neuron 11, 279-289. doi: 10.1016/0896-6273(93)90184-S

Tsodyks, M. V., and Markram, H. (1997). The neural code between neocortical pyramidal neurons depends on neurotransmitter release probability. Proc. Natl. Acad. Sci. U.S.A. 94, 719-723. doi: 10.1073/pnas.94.2.719
Turner, D. A., and West, M. (1993). Bayesian analysis of mixtures applied to post-synaptic potential fluctuations. J. Neurosci. Methods 47, 1-21. doi: 10.1016/0165-0270(93)90017-L

Varela, J. A., Sen, K., Gibson, J., Fost, J., Abbott, L. F., and Nelson, S. (1997). A quantitative description of short-term plasticity at excitatory synapses in layer $2 / 3$ of rat primary visual cortex. J. Neurosci. 17:7926. doi: 10.1523/JNEUROSCI.17-20-07926. 1997

Walmsley, B., Edwards, F. R., and Tracey, D. J. (1988). Nonuniform release probabilities underlie quantal synaptic transmission at a mammalian excitatory central synapse. J. Neurophysiol. 60, 889-908. doi: 10.1152/jn.1988.60. 3.889

Wang, Y., Markram, H., Goodman, P. H., Berger, T. K., Ma, J., and Goldman-Rakic, P. S. (2006). Heterogeneity in the pyramidal network of the medial prefrontal cortex. Nat. Publ. Group 9, 534-542. doi: 10.1038/ nn1670

Williams, L. E., and Holtmaat, A. (2018). Higher-order thalamocortical inputs gate synaptic long-term potentiation via disinhibition. Neuron 101, 1-17. doi: $10.1101 / 281477$

Zucker, R. S., and Regehr, W. G. (2002). Short-term synaptic plasticity. Annu. Rev. Physiol. 64, 355-405. doi: 10.1146/annurev.physiol.64.092501. 114547

Conflict of Interest Statement: The authors declare that the research was conducted in the absence of any commercial or financial relationships that could be construed as a potential conflict of interest.

Copyright (C) 2019 Bykowska, Gontier, Sax, Jia, Montero, Bird, Houghton, Pfister and Costa. This is an open-access article distributed under the terms of the Creative Commons Attribution License (CC BY). The use, distribution or reproduction in other forums is permitted, provided the original author(s) and the copyright owner(s) are credited and that the original publication in this journal is cited, in accordance with accepted academic practice. No use, distribution or reproduction is permitted which does not comply with these terms. 